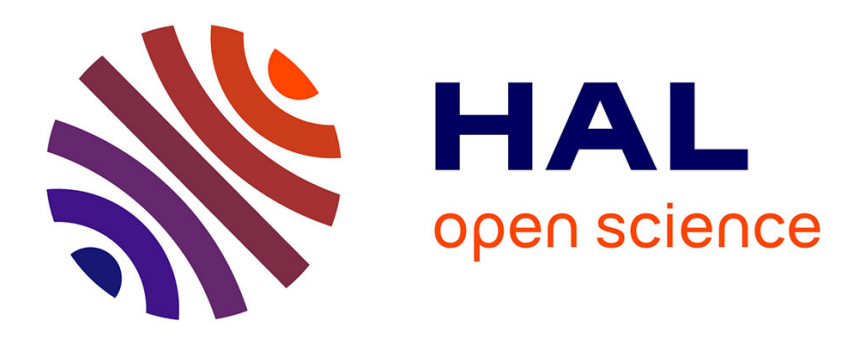

\title{
Prise en charge, commitment ou scène énonciative
}

Denis Paillard

\section{To cite this version:}

Denis Paillard. Prise en charge, commitment ou scène énonciative. Langue française, 2009, 162, pp.109-128. halshs-00746330

\section{HAL Id: halshs-00746330 \\ https://shs.hal.science/halshs-00746330}

Submitted on 27 Nov 2012

HAL is a multi-disciplinary open access archive for the deposit and dissemination of scientific research documents, whether they are published or not. The documents may come from teaching and research institutions in France or abroad, or from public or private research centers.
L'archive ouverte pluridisciplinaire HAL, est destinée au dépôt et à la diffusion de documents scientifiques de niveau recherche, publiés ou non, émanant des établissements d'enseignement et de recherche français ou étrangers, des laboratoires publics ou privés. 


\section{Prise en charge, commitment ou scène énonciative}

Denis Paillard

Laboratoire de linguistique formelle

(UMR 7110)

Université Paris Diderot $\square$ Paris 7

Cet article, partant dune réflexion sur la notion de prise en charge et, dans une moindre mesure, de commitment propose une approche alternative reposant sur la notion de scène énonciative.

\section{Prise en charge / commitment : quelques remarques critiques}

Un énoncé assertif $\mathrm{d}$ un côté renvoie à une prise de position du locuteur, de 1 autre exprime quelque chose à propos d un état de choses du monde (nous laissons pour liinstant de côté la relation locuteur / interlocuteur). Ces deux moments, qui inscrivent lẻnoncé dans une problématique du sujet et une problématique du monde, sont étroitement liés. Les notions de prise en charge et de commitment sont deux approches distinctes de cette question, la prise en charge privilégiant le rapport du sujet à ce qui est dit, le commitment 1adéquation ou, plus précisément, la vérité de ce qui est dit présentée comme un enjeu pour le locuteur.

Dans la définition de la prise en charge que propose A. Culioli (1980:184) la vérité est subjective : « sens technique de prendre en charge : dire ce que l'on croit (être vrai)»" Dans la théorie scandinave de la polyphonie linguistique, la responsabilité est définie en termes de source énonciative et non pas en terme de vérité 2 . Un énoncé est défini comme un «point de vue ». Enfin, on notera que des chercheurs comme Culioli mais aussi Ducrot et, plus récemment, Nølke ${ }^{3}$ ont pris leurs distances avec la notion de prise en charge jugée trop vague et donc peu opératoire.

Les approches utilisant la notion de commitment $\mathbf{s}$ intéressent au locuteur sous 1 angle de son positionnement public à propos de la vérité (fausseté) d un contenu propositionnel donné.

\footnotetext{
${ }^{1}$ Pour une systématisation de cette approche, cf. P. Laurendeau (1989).

${ }^{2}$ Sur ce point, nous renvoyons à 1 article de P. Dendale et D. Coltier (2005).

${ }^{3}$ Cf. Ducrot (1983 : 179) ; Nølke, Table Ronde consacrée à la « prise en charge » lors de la Xème Conférence de $1 \square \operatorname{PrA}$ à Göteborg ( $8 \square 13$ juillet 2007). Pour ces deux auteurs, cette remise en cause est directement liée à la notion de polyphonie, en tant que lieu où se pluralise la notion de sujet. Dans le cas de Culioli, cet abandon est lié à une nouvelle définition de 1 assertion, sur laquelle nous reviendrons.
} 
C. Beyssade et J.M. Marandin (2008) écrivent «Se commettre à une proposition p, c est rendre public le fait qu on est prêt à soutenir que cette proposition est vraie, c est avoir des arguments en faveur de $\mathrm{p}$ ». En dautres termes, la notion de commitment est étroitement associée à une sémantique vériconditionnelle, qui laisse à la pragmatique (avec la théorie des actes de langage) 1ऍessentiel de la problématique du sujet ${ }^{4}$. Avec la notion de commitment la vérité du contenu propositionnel relève d un engagement du locuteur, sans que, pour autant, 1「on puisse parler de vérité subjective telle quelle est mise en place dans les théories énonciatives ${ }^{5}$.

Notre approche critique de ces deux notions repose sur un ensemble de considérations $\mathrm{d}$ ordre théorique mais aussi d ordre empirique.

Sur le plan théorique, prise en charge et commitment, 1un comme 1 autre, privilégient de fait 1 un des deux moments présentés ci-dessus, et, à ce titre, peuvent être considérées comme réductionnistes. La notion de prise en charge, centrée généralement sur une problématique du sujet, tend à relativiser fortement le rapport de ce qui est dit à lêtat de choses quill exprime (pour un linguiste comme Ducrot, dire le monde n est pas un enjeu pour le linguiste); on notera également qu en dehors de la sémantique vériconditionnelle, la notion de « vérité » reste souvent intuitive. Inversement, la notion de commitment associent étroitement la problématique du sujet à celle de la vérité / fausseté de ce qui est dit $^{6}$. Nous considérons que les rapports qui se jouent entre un sujet, un contenu propositionnel et le monde sont éminemment variables et doivent être calculés.

Ci-dessous, en nous limitant aux énoncés assertifs, nous présentons brièvement quatre types de données afin dilllustrer la complexité des phénomènes en jeu. Selon nous, la prise en compte de ces données débouche sur un questionnement de la notion de sujet comme individu $\square$ locuteur, mais aussi de la sémantique vériconditionnelle (dans une assertion le contenu propositionnel ne se réduit pas à une problématique vrai / faux ().

(a) Soit les deux énoncés :

(1a) Paul est un enfant / (1b) Paul est fatigué

\footnotetext{
${ }^{4}$ On notera que la plupart des chercheurs (Culioli, Ducrot, Nølke) qui, à un moment donné, ont utilisé la notion de prise en charge, récusent la coupure stricte entre sémantique et pragmatique.

${ }^{5}$ Beyssade et Marandin (2008) proposent dẻlargir la notion de commitment à des énoncés autres qu âssertifs.

${ }^{6}$ Nous sommes conscients du caractère quelque peu abrupt de ces formulations ; pour une critique plus détaillée tant de la théorie de Ducrot que de la sémantique vériconditionnelle, le lecteur pourra se reporter à un article déjà ancien écrit avec S. De Vogüe (De Vogüe \& Paillard, 1987).
} 
Dans (1a) 1 individu Paul est présenté comme une occurrence de la propriété «être enfant» (prise ou non au sens littéral). Le locuteur catégorise Paul, en le ramenant (le temps de son énoncé) à la propriété quiil en prédique. Dans (1b) 1๔enjeu est différent : il s agit d arriver à dire non pas 1 iindividu mais un «mode de présence » actualisé de cet individu : le rapport du locuteur au monde est profondément différent : il se présente comme travail d expertise ou encore de témoignage sur un état de fait concernant $\mathrm{Paul}^{7}$. L'enjeu n'est plus de rapporter le monde à la langue (à une propriété), mais d'aligner le dire sur le monde. Dans cet alignement, c'est le dire qui se trouve déstabilisé, visant à se conformer à ce monde qu'il cherche à dire.

(b) La séquence Il pleut $\mathrm{n}$ a pas le même statut dans :

(2a) A - Quel temps fait-il ? B. $\square$ Il pleut

(2b) A $\square$ Et si on allait se promener... B. $\square$ Il pleut

Dans (2a) Il pleut est un énoncé catégorisant dont 1adéquation à 1 êtat de choses qu ill décrit peut être questionnée. Dans (2b) Il pleut est un point de vue ${ }^{8}$ opposé à un premier point de vue (1Æenvie daller se promener formulée par A). Quant à 1 êtat de choses en question ${ }^{9}$ il se rejoue $\mathrm{d}$ un point de vue à 1 autre.

(c) La présence d un ou plusieurs marqueurs discursifs tend à marginaliser, relativiser ou encore moduler 1 implication du sujet.

(3) A. $\square$ Prideš ? B. (a) Pridu / (b). Da už pridu $\left(\right.$ russe $^{10}$ )

A. Tu viens ? B. Je viens

(a) est une réponse positive à la question «venir / ne pas venir », (b) (avec une réalisation phonétique réduite de $d a u z ̌$ ) et un accent contrastif sur pridu est une réponse positive mais où le locuteur signifie quiil ne peut pas ne pas venir (si cela ne tenait quà lui il ne viendrait pas). Cette réponse traduit une position complexe du locuteur entre résignation $\square$ capitulation et résistance.

d. Certaines séquences réduites à un adverbe - mot du discours ${ }^{11}$ sont interprétées comme des réponses (affirmatives) à une question, en concurrence avec oui :

\footnotetext{
${ }^{7}$ Ceci peut être mis en rapport avec la distinction faite par G.N. Carlson (1978) entre prédicats épisodiques et prédicats dindividus.

${ }^{8}$ « Point de vue » n est pas pris ici au sens que lui donne Ducrot ou encore Nølke : chez ces auteurs, ce terme tend à désigner tout type d ênoncé, alors que pour nous il désigne un certain type deenoncé (cf. ci-dessous).

${ }^{9}$ Le terme «état de choses » est peu satisfaisant et pris faute de mieux pour désigner le monde tel quil est convoqué par lënoncé. D une certaine façon leêtat de choses est «ce dont on parle en disant ce que lon dit» (cf. la notion daboutness).

${ }^{10}$ Dans cet article, pour illustrer certains points (concernant les marqueurs discursifs) nous serons amenés à prendre des données du russe.
} 
(4) $\square$ Tu seras à la réunion? - Oui / bien sûr / certainement / nécessairement / effectivement / forcément / naturellement / probablement / malheureusement / etc. ${ }^{12}$

Par exemple, forcément associe la présence annoncée du locuteur à une raison contraignante $^{13}$; naturellement convoque 1 ○rdre des choses, ce qui revient à relativiser la dimension dẻvénement singulier engageant le sujet (la venue est présentée non pas comme une décision mais comme inscrite dans 1 ordre des choses); avec malheureusement, la présence est assimilée à un «malheur». On peut considérer que ces différents marquerus signifient que pour ce qui est de laffirmation de sa présence, le locuteur se met en retrait.

Ci-dessous nous proposons un cadre qui, permet, nous semble-t-il, permet de rendre compte de façon intégrée et de la place du sujet et du rapport, éminemment variable, dun énoncé à 1 êtat de choses quill exprime. Dans notre approche, la notion clef est celle de scène énonciative. Elle repose sur 1hypothèse que 1ênonciation n est pas 1َacte $\mathrm{d}$ un sujet qui produit un énoncé mais un processus qui peut être reconstitué à partir de 1َagencement des formes qui composent un énoncé. L ênonciation est donc 1 ensemble des déterminations (dont les formes qui la constituent sont les marqueurs) qui interviennent dans la production de 1ênoncé. Ni le sujet ni le monde ne sont posés comme premiers, dans un rapport d extériorité à 1 ênoncé lui-même : ils ne sont pris en compte quà travers ce qu en dit 1eênoncé ${ }^{14}$.

\section{Le dire et le dit $^{15}$}

Le dire et le dit, titre d un ouvrage de O. Ducrot (1984), postule une séparation nette entre le dire comme processus et son résultat (ce qui est dit : 1eenoncé) : d un côté le locuteur

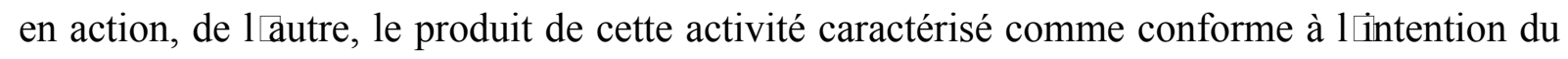
locuteur $^{16}$. L activité est en amont de leenoncé, espace pré-linguistique déployé par la seule grâce d un sujet tout puissant, ayant un objet à dire, un projet de dire et développant en toute liberté sa stratégie visant à mettre en mots ce quill a en tête. Notre conception de 1ênonciation, évoquée ci-dessus, signifie une remise en cause de cette séparation.

\footnotetext{
${ }^{11}$ Nous reviendrons en 4 sur le statut de ces unités comme « mots du discours ».

${ }^{12}$ A noter également, la combinaison Oui, bien sûr / oui, naturellement.

${ }^{13}$ Sur forcément, se reporter à 1 article de Danjou-Flaux et Gary Prieur (1993). Ajoutons que selon la place de forcément la même séquence change de statut; comparer : (a) il est forcément là / quelque part (cherche-le) et de (b) Forcément, il est là / *quelque part.

${ }_{14}^{14}$ Sur cette lecture de la théorie de Culioli, cf. De Voguë (1992), Franckel \& Paillard (1999), Paillard (2006b).

${ }^{15}$ Ce paragraphe doit beaucoup à une réflexion menée en commun avec Sarah de Voguë.

${ }^{16}$ Notre propos ici n est pas de discuter la théorie de Ducrot. Sur ce point, cf. De Voguë \& Paillard (1987).
} 
Lorsque 1 1 on s interroge sur ce que désigne le verbe dire, on constate qu en fonction de la nature de ses compléments, il peut signifier " proférer des mots », « exprimer un état de choses » et «affirmer un contenu ». A travers ces trois acceptions sont convoqués les trois paramètres de base de la scène énonciative: les formes de la langue qui constituent la matérialité de 1eênoncé, 1eetat de choses qui en tant qu êtat du monde est le «à dire » et, enfin, le «contenu» qui, dans une première approximation, désigne ce que les mots disent du monde. En fait, le contenu renvoie non seulement à ce que 1ểnoncé dit mais aussi à un « vouloir dire » qui ne se confond pas avec le « effectivement dit». Un indice de cette noncoïncidence apparaît dans les expressions du type : (5) Tu vois ce que je veux dire par là ? (6) Je vois / ne vois pas ce que tu veux dire, .... Dans ces énoncés, centrés sur un ajustement entre deux locuteurs concernant ce qui est dit, la vérification met en avant le « vouloir dire » et non 1ênoncé lui-même (cf. 1 lïmpossibilité des deux séquences avec simplement le verbe dire: $(5 \mathrm{a}) *$ Tu vois ce que je dis ? et (6a) *Je vois / ne vois pas ce que tu dis...). D une certaine façon, dans ces énoncés il y a deux contenus : celui du dit, dune part, celui correspondant au vouloir dire, dautre part. Dans (5) et (6) il $\mathrm{n}$ y a pas nécessairement coïncidence entre les deux. Mais ce contenu correspondant au «vouloir dire » n a pas de réalisation autonome, il n est présent quà̀ travers les mots de 1 eênoncé (désignés par là dans (5) ci-dessus), et en même temps, rien ne garantit que les mots en jeu réussissent à dire ce « vouloir dire ». Bien plus, réussite ou échec tendent à échapper au locuteur (cf. je n'arrive pas à le dire). Dans Frantext, on trouve un très grand nombre d exemples (plusieurs centaines) avec je veux dire.

Si 1 $\not$ on s interroge sur le statut de ce «vouloir dire », il apparaît quill ne relève pas dune pure intentionnalité qui serait le projet du locuteur; plus exactement, il est nécessaire de distinguer deux vouloir dire : un vouloir dire désignant ce projet et un vouloir dire qui est constitutif du contenu. Dans 1 expression «quand je dis ça $a_{1}$, je veux dire ça $a_{2}$, je veux dire peut $\mathrm{s}$ interpréter comme désignant le projet (dans ce cas on peut intercaler en réalité et ça désigne alors un autre dire $\square$ et donc un autre contenu), mais aussi comme désignant le contenu (dans ce cas $c ̧ a_{2}$ relève d une reformulation, avec le recours à d autres mots pour dire ce même contenu : il relève du même vouloir dire). Nous donnons ci-dessous quelques exemples tirés de Frantext :

(7) Quand je dis que je l'ai mis en pièces, précisa-t-il par amour de la vérité littérale, je veux dire que je l'ai déchiré en deux (J. Green, Moira, tiré de Frantext)

(8) Vers onze heures, elle prend la physionomie d' une bonne grand' mère... il y a des jours où je me dis que j' aurais voulu savoir parler, et quand je dis parler, je veux dire assembler des 
mots de manière à fermer la bouche à mes adversaires ou à la leur faire ouvrir d' étonnement, enfin briller, convaincre (J. Green, Journal T.2, tiré de Frantext)

(9) Quand je dis " elle les a eus ", je veux dire plutôt qu'elle s'était emparée de leur esprit et qu'ils dépendaient d' elle, qu' elle sentait qu' elle pouvait les faire agir, vous comprenez ? (A. Maurois, Climats, tiré de Frantext)

Dans (7) et (8) il s agit dun travail de reformulation $\square$ explicitation dun même vouloir dire qui se réalise par deux séquences successives. En (9) la présence de plutôt (que 1ळon peut remplacer par en fait, en réalité) tend à signifier quill ne sagit pas d une simple explicitation, mais du passage à un autre projet : il y a discontinuité entre les deux séquences.

Pour distinguer le vouloir dire projet $\square$ du vouloir dire contenu $\square$ nous noterons le premier « vouloir dire » (dans ce cas, le verbe dire commute avec n importe quel autre verbe : c est lintention du locuteur qui est en jeu) et le second « vouloir + dire » en tant que formant un bloc indissociable. Le « contenu » n est plus simplement ce que les mots formant 1eênoncé disent du monde ; il est redéfini comme 1 association d un vouloir + dire et des formes qui composent 1 ểnoncé, le vouloir + dire excédant par définition le effectivement dit $\square$ comme le montre, en particulier, le travail de reformulation $\square$ explicitation associant plusieurs énoncés à un même vouloir + dire. Nous proposons de désigner cette association d un $\downarrow$ vouloir + dire $\square$ et $\mathrm{d}$ un ou plusieurs énoncés par le mot dire pris comme substantif.

Dans cette perspective, le vouloir + dire se révèle au fil du discours, et le locuteur (et non pas seulement 1 linterlocuteur), découvre ce vouloir + dire au fur et à mesure de son discours. Ceest 1 ênoncé (ce qui est dit) dans sa matérialité qui convoque le vouloir + dire en tant que tel inaccessible et en même temps, 1 ẻnoncé ne se confond pas avec ce vouloir + dire comme en témoigne le travail de reformulation toujours possible (d une certaine façon aucun énoncé n êpuise le vouloir + dire, si ce n est par défaut).

Ce dire est toujours un dire parmi d autres. Il n est jamais que la perception / représentation du «à dire» (le monde) qui en tant que tel n est pas de 1 ørdre du formulable (il y a une discordance irréductible entre le dire et le monde). Dire un état de choses du monde en tant que «à dire » revient à donner une forme à cet état de choses. $\mathrm{Ce}$ travail de façonnage est matérialisé dans un énoncé, mais cet énoncé, par définition, ne peut être que partial (il renvoie à une perception / représentation du monde par un sujet) et partiel : il ne peut quêchouer à dire le « à dire » jusqu au bout.

Enfin, si le dire n est jamais qu un dire parmi d autres, cela tient à ce que d autres mots sont possibles et que rien ne garantit a priori $1 \varangle$ adéquation » des mots utilisés : dune 
certaine façon, les mots échappent au locuteur car ils disent « ce quills veulent dire » et rien ne dit que liinterlocuteur, dans son travail d interprétation, interprète ce vouloir dire des mots comme le locuteur.

Nous proposons donc de définir un dire comme une façon partiale et partielle d'exprimer par un énoncé un état de choses du monde.

\section{La notion de scène énonciative}

Dans un article récent, Culioli (2001) a proposé une définition de 1assertion qui se distingue de façon radicale de celle qu iil a donnée en 1980 et qui était centrée sur la notion de prise en charge. Cette nouvelle définition est la suivante : " je tiens à parler et à dire (= rendre public $^{17}$ ) que je pense / crois / sais que 'p est le cas'». Cette définition articule deux moments en les distinguant nettement :

- une première partie convoque $j e$, le sujet du dire : «je tiens (à parler ${ }^{18}$ et) à dire $(=$ rendre public) que je pense / crois / sais que [ ]». Quatre points nous paraissent devoir être soulignés :

a. « je tiens » c est $s$ 'engager, au sens de prendre un risque ; cet engagement $\mathrm{n}$ est pas synonyme de la notion de commitment (centrée, nous 1 avons vu, sur le rapport du locuteur à la vérité du contenu propositionnel). Pour Culioli (en écho à la notion de force assertive chez Frege) cela signifie uniquement que ce qui est dit importe au locuteur (on est en deçà de toute problématique de la vérité) ;

b. " parler» renvoie au fait de proférer des sons articulés et de chercher à établir par ce moyen une relation à un autre sujet (rien ne dit que cette relation à 1 autre soit acquise $\left.^{19}\right)$

c. le fait de rendre public $\mathbf{p}$ pose le problème de 1autre que moi, au sens où je prends le risque d être incompris ou encore contredit ;

d. le rapport du locuteur à ce quill dit est variable, allant dune pure subjectivité ([je pense $\$ ) à une vérité établie ( je sais $\square$ ) en passant par une vérité subjective ( je crois $\square$ ).

\footnotetext{
${ }^{17}$ Rappelons que dire correspond à une racine indo-européenne signifiant « montrer ».

18 «parler» ne figure pas dans le texte de 1 article mais a été introduit par A. Culioli à différentes reprises lors de son séminaire à 1ENS Ulm (2007 $\square 2008)$.

19 Rappelons la phrase de Culioli : «La compréhension est une forme particulière de malentendu »). Linterlocution c est aussi la capacité de la refuser ou de linterrompre, ou encore la possibilité de déformer 1๔espace du dialogue ou de rechercher 1 ăaccord sur un point autre que celui présenté au départ. La duplicité, 1 acte manqué, le mensonge sont constitutifs de la communication et ne sauraient être ramenés à des cas de déviation.
} 
La subjectivité se manifeste ici sur trois plans : le fait même de parler et de rendre public quelque chose qui mimporte, la mise en place $\mathrm{d}$ un espace intersubjectif et, enfin, la modalité portée par penser, croire et savoir ». Si 1ळon retrouve ici certains termes présents dans la définition de commitment proposée par Beyssade et Marandin, il faut insister sur le fait que leur statut n est pas le même.

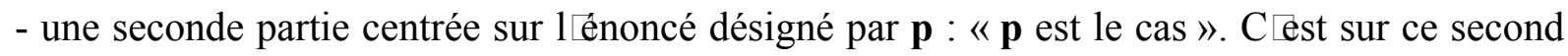
point que le déplacement par rapport à la définition de (1980) est le plus net : abandon de la notion de « vrai » et introduction de la notion de calcul. En effet, dans 1 article cité, «p est le cas » est présenté comme le produit d un calcul débouchant sur la sélection d une séquence $\mathbf{p}$ en relation avec lêtat de choses du monde à dire ( (ce qui est le cas $\square$ ). Il y a calcul dans la mesure où la séquence $\mathbf{p}$ est sélectionnée parmi dautres séquences a priori possibles, ce que Culioli note $(\mathbf{p}, \mathbf{p})$ : au départ il $\mathrm{n} \bigvee \mathrm{y}$ a pas de rapport nécessaire entre $\mathbf{p}$ et le à dire $\square$ La prise en compte de $\mathbf{p}$, représentant les séquences possibles autres que $\mathbf{p}$, inscrit dans le dispositif même la possibilité, dans le cadre dun enchaînement discursif, dun travail de reformulation / explicitation par le recours à un autre énoncé, que cette reformulation soit délibérée ou au contraire témoigne de la difficulté (plus ou moins maîtrisée) qu ill y a à arriver à dire ce qu ill y a à dire. Culioli représente le calcul débouchant sur « $\mathbf{p}$ est le cas » par le schéma suivant :

\section{$\mathbf{p}, \mathbf{p} \square($ est le cas $)$}

\section{$\rightarrow$ p est le cas}

\section{(quelque chose) est le cas}

La première ligne $\mathbf{p}, \mathbf{p} \square$ (est le cas) correspond au niveau des formes linguistiques : « est le cas » signifie qu en parlant le locuteur va sélectionner $\mathbf{p}$ ou $\mathbf{p} \square$; la ligne quelque chose est le cas désigne le monde en tant que «à dire»: comme nous 1َavons souligné ci-dessus, «quelque chose» signifie qu en tant que tel le monde $n \bowtie$ pas de forme. p est le cas s interprète comme $\mathbf{p}$ est la séquence de la langue (1ểnoncé) associée à ce qui « est le cas », c'est-à-dire 1 êtat de choses du monde en tant que «à dire ». D où cette glose de « p est le cas » : « la séquence $\mathbf{p}$ est sélectionnée en tant qu elle rend compte de ce qui est le cas $\square$ ».

La sélection de $\mathbf{p}$ s inscrit dans un espace « intersubjectif » que Culioli organise autour de trois positions ${ }^{20}$ :

\footnotetext{
${ }^{20}$ Rappelons, dans le prolongement de ce qui est défendu en 2. que, dans notre approche, la problématique du sujet n est pas une problématique des individus (locuteur / interlocuteur), c est la raison pour laquelle nous
} 
So et S1 désignent deux positions dans un rapport daltérité (la position S1 est seconde par

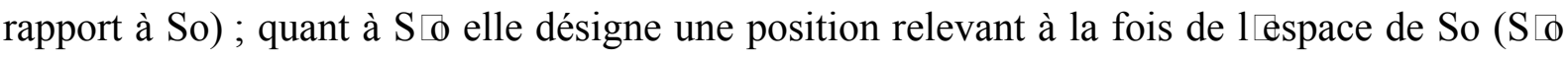
est 1 ilmage en miroir de So) et de celui de $\mathrm{S} 1$ ( $\mathrm{S} \triangleright$ est alors la représentation que se fait So de $\mathrm{S} 1)^{21}$. Les recherches dun phonéticien russe (S. Kodzassov, (2003)) ont mis en évidence pour le russe une prosodie dite d厄emphase et une prosodie quill nomme " anti-emphase ». La première caractérise les énoncés où la sélection de $\mathbf{p}$ constitue un enjeu (ce qui est le cas de 1 âssertion simple ; la position S1 n est pas actualisée mais représente la position à partir de laquelle peut se mettre en place la contestation de $\mathbf{p}$ comme disant « ce qui est le cas »), la seconde, les cas où la sélection de $\mathbf{p}$ n est pas / plus un enjeu (ce qui recouvre des valeurs pragmatiques diverses allant de la résignation-capitulation à la complicité). Nous proposons de rendre compte de ces variations prosodiques en associant $\mathbf{p}$ à telle ou telle position de 1 espace intersubjectif: avec une prosodie de type emphase, $\mathbf{p}$ est mis en relation avec la position So ; avec une prosodie anti-emphase, p est associé à la position S1 et présenté comme « hors enjeu». Quant à la position $\mathrm{S} \_$elle correspond aux cas où la prosodie ne relève pas de la distinction emphase / anti-emphase.

Dans cette perspective, (3b) A. $\square$ Prideš ? B. Da už pridu (A. Tu viens ? B. Je viens) peut être caractérisé comme une assertion activant deux positions : la prosodie du type anti-emphase associe $\mathbf{p}$ à la position S1 (capitulation $\square$ résignation : «venir » est la position de 1 autre). Et 1 accent contrastif, qui porte sur pridu signifie que $\mathbf{p} \square$ (« ne pas venir »), associé à la position So, est opposé à $\mathbf{p}$ (résistance au sens d un maintien $\mathrm{d}$ une double altérité : altérité $\mathbf{p} / \mathbf{p} \square$ et altérité des positions So et $\mathrm{S} 1$ ).

Dans le prolongement de cette conception de 1 assertion on peut proposer une définition de liinjonction, de la question et de 1 exclamation combinant sélection et / ou nonsélection d une séquence et des positions subjectives ${ }^{22}$.

Pour l'injonction (telle quelle est réalisée par la forme limpératif $\square$ du verbe dans des langues comme le français ou le russe $)^{23}$, 1hypothèse est la suivante : 1 i̇mpératif signifie que

parlons de «positions » (comparables dans une certaine mesure aux « voix » introduites par Ducrot). So, S1 et $\mathrm{S} \curvearrowleft$ désignent donc non pas des individus mais des positions articulées à la sélection de $\mathbf{p}$ ou $\mathbf{p}$

${ }^{21}$ Un même énoncé peut mettre en jeu plus dune position : cf. la représentation de 1 i̇mpératif ci-dessous ainsi que celle proposée pour $3 \mathrm{~b}$.

${ }^{22}$ Précisons que cette typologie est fondée sur une représentation des formes linguistiques convoquant des positions énonciatives et ne fait pas appel à la notion dacte illocutoire mettant en jeu des individus. Elle permet un calcul permettant de rendre compte des différentes interprétation des énoncés assertifs, injonctifs, interrogatifs et exclamatifs. 
la relation prédicative est représentée comme associée à deux positions subjectives, chaque position correspondant à un statut particulier du procès désigné par la forme impérative : $\mathrm{d}$ un côté, le procès est visé : position So correspondant à la sélection de la valeur positive du procès, de 1 autre, le procès est en attente de validation : position S1 correspondant à la nonsélection dune valeur, non sélection qui peut déboucher sur la sélection d une valeur $\mathrm{du}$ procès (p si S1 rejoint So ou $\mathbf{p} \square$ si S1 valide un autre procès que p) mais qui peut aussi se maintenir comme telle ( $\mathrm{S} 1$ ne réagit pas). Ce que 1ळon peut représenter (en notant la valeur positive du procès par $\mathbf{p}$ et la non-sélection $\mathrm{d}$ une valeur $\operatorname{par}(\mathbf{p}, \mathbf{p})$ :

$$
\text { So : } \mathbf{p}
$$

\section{$\mathrm{S} 1: \mathbf{p}, \mathbf{p}$}

Dans ce schéma, la position $\mathrm{S} 1: \mathbf{p}, \mathbf{p} \square$ est un pôle dindétermination; on est en deçà de la sélection d une valeur que ce soit $\mathbf{p}$ ou $\mathbf{p} \square(\mathbf{p} \square$ correspond à un refus de valider $\mathbf{p}$ ou encore à la validation d un procès autre que $\mathbf{p}$ ) face à la visée de $\mathbf{p}$ (position de So).

Cette approche de 1 ìmpératif diffère de celle qui considère 1 injonction comme un acte de langage du type « commande », convoquant le locuteur et 1 interlocuteur. Ceest liimpératif en tant que forme du verbe qui construit ces deux positions. Dans le cadre de cette hypothèse, il est possible de rendre compte et des valeurs injonctives et des valeurs non injonctives (narration, concession, hypothétique) de liimpératif dans une langue comme le russe (Paillard, 2008) ou encore, de façon moindre, le français (cf. Approche et je tire). L existence dans de nombreuses langues de ces valeurs renforce la nécessité de distinguer ces deux positions du couple « locuteur / interlocuteur ».

Toujours dans cette perspective, on peut définir la question comme associant So à $(\mathbf{p}, \mathbf{p})$ (non sélection, mais avec pondération éventuelle sur $\mathbf{p}$ ou $\mathbf{p}$ ) et $\mathrm{S} 1$ à la sélection possible (mais non nécessaire) de $\mathbf{p}$ ou de $\mathbf{p} \square$ Enfin 1 exclamation peut être caractérisée par le fait que $\mathbf{p}$ est hors altérité (il ne relève pas de 1Ґalternative $\mathbf{p} / \mathbf{p}$ ) et que 1๔espace intersubjectif

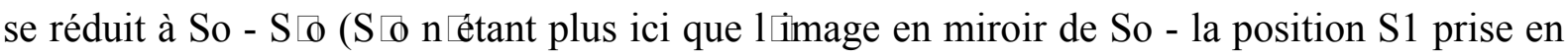

\footnotetext{
${ }^{23}$ Nous reprenons ici les hypothèses formulées dans un article consacré à l limpératif en russe (Culioli \& Paillard, 1987).
} 


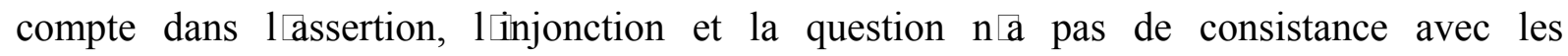
exclamatives).

Dans les sections 2 et 3, partant de 1 lidée que c est 1 lênoncé, à travers les agencements de formes qui le constituent, qui met en scène les conditions de sa production, nous avons progressivement dégagé les principaux éléments qui participent de ce que nous appelons la scène énonciative. La notion de dire telle qu elle a été introduite ci-dessus (« un dire est une façon partiale et partielle d exprimer par un énoncé un état de choses du monde ») définit le cadre et les limites de cette prise en compte, limites que soulignent les termes «partiale » et « partielle ». Enfin, la sélection (non-sélection, sélection en attente) dune séquence linguistique en relation à un état de choses est le résultat dun calcul, indissociable de liinscription de la séquence en jeu dans un espace intersubjectif structuré autour de trois positions.

En résumé, la notion de scène énonciative en tant que représentation de ce que nous appelons un dire est un dispositif qui tente de dépasser 1 lextériorité première entre le locuteur - individu et son énoncé, entre lênoncé et lêtat de choses quiil entend exprimer, entre le locuteur et lïnterlocuteur. Ceest la matérialité de 1 lẻnoncé en tant quagencements de formes qui permet de reconstituer et les positions subjectives en jeu, et le statut de leênoncé en tant que donnant une certaine forme (par définition partiale et partielle) à un état de choses du monde. La scène énonciative est un espace dynamique où les positions peuvent se rejouer dans un travail d explicitation / reformulation du vouloir + dire.

En 4. nous montrons la place qu occupent différents types de marqueurs discursifs dans la construction du dire.

\section{Marqueurs discursifs ${ }^{24}$}

La majorité des travaux concernant les marqueurs discursifs accordent une large place à la pragmatique. Ce qui, selon nous, doit être mis en relation avec une autre constatation : dans la plupart des approches, les marqueurs discursifs ne sont pas considérés comme formant une classe dunités de la langue identifiée par un ensemble de propriétés : le terme de mots du discours introduit par O. Ducrot souligne cette extériorité au système de la langue. Cette pragmatique dominante explique le très grand morcellement des approches, la prolifération des terminologies et 1utilisation $\mathrm{d}$ un même terme pour désigner des phénomènes

\footnotetext{
${ }^{24}$ Pour une discussion plus détaillée des marqueurs discursifs en relation avec la scène énonciative, on peut se reporter à Paillard (2008, à paraître).
} 
radicalement différents. La vingtaine de contributions à 1ळuvrage Approaches to discourse particles (K. Fisher, 2006) réunit des approches profondément divergentes sur de nombreux points. La difficulté quill y a à formuler des critères permettant didentifier une ou plusieurs classes de marqueurs discursifs s explique, en partie, par le fait que, dans des langues comme le français ou le russe, la plupart des mots ou locutions assimilés à des marqueurs discursifs ont une autre appartenance catégorielle dans la langue (nom, forme verbale, adjectif, adverbe). Bien plus, le redéploiement discursif de telle ou telle unité ne semble permettre aucune généralisation : vraiment a un statut de marqueur discursif, faussement non. La « cristallisation » de telle ou telle unité comme marqueur discursif apparaît comme largement contingente. Cela n est pas sans rapport avec le fait qu un très grand nombre dẹtudes traitent dun seul marqueur, pris dans sa singularité.

Sur la base de 1êtude détaillée dune cinquantaine de marqueurs discursifs du russe ${ }^{25}$ et du français, nous considérons les marqueurs discursifs comme formant une classe d'unités de la langue qui ont une sémantique (que nous qualifierons de discursive) et une distribution $^{26}$. De plus, à 1 iintérieur de cette classe, il est possible de distinguer plusieurs sousclasses, les unités de chaque sous-classe se caractérisant par une sémantique et des propriétés distributionnelles spécifiques ${ }^{27}$. Ci-dessous, nous présentons trois sous-classes, en montrant comment leur sémantique intervient dans la construction de la scène énonciative :

- les mots du discours (MD) : ils spécifient à quel titre une séquence ${ }^{28}$ correspondant à leur portée est « une façon partiale et partielle d exprimer un état de choses »; (cf. d'ailleurs, quand même, effectivement, vraiment).

- Les particules énonciatives ${ }^{29}$ : elles spécifient $\mathbf{p}$ du point de vue de son rapport à $\mathbf{p} \sqsubset$; (cf. už en (3b), bien dans on achève bien les chevaux).

\footnotetext{
${ }^{25}$ Leétude (dans le cadre d un programme russe $\square$ français) des marqueurs discursifs du russe a donné lieu à trois recueils collectifs, plusieurs thèses et de nombreux articles.

${ }^{26}$ En posant que les marqueurs discursifs forment une classe d unités de la langue nous défendons la thèse quilil est possible de les définir non seulement par leur fonction mais aussi par ce quills sont. Sur ce point cf. Paillard (1998, 1999, et 2008, à paraître).

${ }^{27} \mathrm{Ce}$ sont ces propriétés distributionnelles en relation à leur sémantique propre qui fondent lidentification des sous-classes de marqueurs discursifs. Pour les mots du discours, cf. Paillard (2006) et Franckel \& Paillard (2006).

${ }^{28}$ Nous parlons de séquence pour désigner la portée du marqueur. La portée d un marqueur discursif $\mathrm{n}$ 厄est pas nécessairement un énoncé : il peut sagir dun mot (Nous avons rencontré un prof, sympa d'ailleurs), dun syntagme (pas question de faire une architecture de banlieue aux Halles, ni en banlieue d'ailleurs, ex. repris de Ducrot), une proposition enchâssée (les enfants, qui d'ailleurs tombaient de sommeil, refusèrent d'aller se coucher), etc. Mais dans tous les cas, le marqueur confère à sa portée un statut discursif spécifique en accord avec sa sémantique.

${ }_{29}$ L ajout du qualificatif «discursives» prend en compte le fait que dans certaines langues, le terme « particules » est employé pour désigner des unités très diverses du point de vue de leurs propriétés.
} 
- les mots du dire : au départ, il s agit de marqueurs discursifs comportant le verbe dire (cf. pour ainsi dire, disons, etc.). Ci- dessous, nous proposons délargir cette classe aux marqueurs discursifs qui actualisent une distance entre un vouloir dire (subjectif, des mots, du monde) et leênoncé en jeu.

Dans (Paillard, 2008, à paraître) nous introduisons une quatrième sous-classe de marqueurs discursifs (désignés comme des «modalisateurs ») qui marquent une forme de brouillage du rapport entre p et « ce qui est le cas » (cf. en français en quelque sorte, genre ; en anglais like; pour le français, un inventaire systématique reste à faire).

Nous sommes conscients de la très grande hétérogénéité des termes employés pour désigner les différentes sous-classes. La terminologie actuelle est le reflet du cheminement qui nous a conduit à distinguer ces différentes sous-classes: en 1eêtat, il saagit de simples étiquettes adoptées à un certain moment de notre recherche. Il sera nécessaire daarriver à une terminologie plus cohérente.

Lintégration des marqueurs discursifs dans le dispositif présenté en 3 permet de dépasser 1 extériorité pragmatique qui caractérise un grand nombre $\mathrm{d}$ êtudes, et de déterminer leur apport à la construction de la scène énonciative. Comme nous 1 avons vu avec les exemples, certains marqueurs discursifs relativisent ou encore modulent la part " subjective » de la valeur référentielle de lênoncé où ils apparaissent. Ci-dessous, nous donnons quelques indications supplémentaires sur les marqueurs discursifs des trois sous-classes et leur sémantique.

\subsection{Les mots du discours}

Les mots du discours spécifient à quel titre la séquence $\mathbf{p}$ correspondant à sa portée est une façon partiale et partielle d exprimer un état de choses R. Nous distinguons deux grands types :

A. MD point de vue : $\mathbf{p}$ est pertinent pour exprimer $\mathbf{R}$ (p est dans une relation possible à R) ;

B. MD adéquation : $\mathbf{p}$ rend compte de $\mathbf{R}$ dune certaine façon / à un certain degré (p est dans une relation forte à $\mathbf{R}$ : il le formate) ;

La description d un MD se fait en deux temps : (a) dabord déterminer de quelle sémantique (point de vue $v s$ adéquation) il relève ; (b) sur la base du contenu lexical du terme pris comme $\mathrm{MD}$, déterminer la façon dont le MD incarne cette sémantique. 


\section{A. MD point de vue.}

La notion de point de vue est une notion éminemment plurielle : sur un même état de choses ou une même situation, il peut y avoir une accumulation de points de vue (cf. considérer une situation de différents points de vue), mais aucun point de vue ne peut prétendre exprimer 1 êtat de choses de façon exhaustive. Cette dimension plurielle signifie qu une séquence « point de vue » est dans une relation faible à lêtat de choses en question : il est simplement posé comme « ayant à voir avec 1 êtat de choses » (c est en ce sens que nous utilisions plus haut le terme de pertinent). La notion de point de vue est prise ici dans une acception distincte de celle quelle a chez Ducrot et chez Nølke : chez ces deux auteurs, la notion de point de vue a une forte dimension subjective et tend à s appliquer à tous les énoncés (sur la notion de point de vue chez Ducrot et Nølke, on peut se reporter à Dendale \& Coltier, 2003). Dans notre approche, un point de vue est partiel et ne peut prétendre à dire 1êtat de choses « jusqu âu bout» : il est toujours confronté à un ou plusieurs autres points de vue.

(10) Il semble très accommodant (q). En réalité, il est d'un sectarisme incroyable (p). (11) - As-tu voté aux présidentielles? - Non (q), d ailleurs je ne vote jamais (p).

Dans (10) 1 altérité des points de vue relève de 1Ґopposition « apparence » / « réalité », et le second point de vue corrige et même disqualifie le premier : en réalité, il est d'un sectarisme incroyable est présenté comme exprimant la « réalité » de $\mathbf{R}$ que rate le premier point de vue qui s en tient aux apparences. Dans (11), je ne vote jamais a pour fonction de prévenir une interprétation de la réponse non comme signifiant «c est une abstention occasionnelle » ou encore «c est parce que c était les présidentielles $\|^{30}$. Non correspond à un premier point de vue qui échoue à dire pleinement ce que signifie pour So le fait de ne pas avoir voté. A la différence de non compatible avec différentes interprétations, d'ailleurs caractérise « ne pas voter » comme hors variation, permettant, par anticipation, de bloquer toute interprétation fautive du non, où $\mathrm{S} 1$ se démarquerait du sens que So entend donner à ne pas être allé voter.

De façon plus générale, un MD point de vue a une double fonction :

(a) il spécifie la séquence $\mathbf{p}$ correspondant à sa portée comme un point de vue, distinct $\mathrm{d}$ un premier point de vue q sur le même état de choses (la séquence correspondant au premier

\footnotetext{
${ }^{30}$ Sur d'ailleurs, cf. Franckel \& Paillard 1997. Dans cet article notre analyse de d'ailleurs est comparée à celle de Ducrot et à celle proposée par leécole de Genève dans le cadre de la théorie de la pertinence.
} 
point de vue ne devient point de vue que de façon rétroactive, avec 1iintervention de la séquence MD p);

(b) conformément à son contenu lexical, il spécifie à quel titre $\mathbf{p}$ est un point de vue distinct du premier : 1 iintroduction d un second point de vue complète / corrige / disqualifie le premier point de vue. Quelle que soit 1aaltérité entre $\mathbf{p}$ et $\mathbf{q}, \mathbf{p}$ est toujours dans un rapport « cumulatif » avec $\mathbf{q}$ tout en étant présenté comme le point de vue de référence.

\section{B. MD adéquation}

La sémantique de 1 adéquation signifie que le MD spécifie de quelle façon et / ou dans quelle mesure la séquence $\mathbf{p}$, portée du MD, rend compte de $\mathbf{R}$. A la différence des MD points de vue, une séquence avec un MD adéquation n est pas un dire parmi d autres : a priori, une telle séquence ne peut être quen concurrence avec une autre séquence pour ce qui est de rendre compte de R. Sur la base de son contenu lexical propre le MD spécifie ce qui est le support

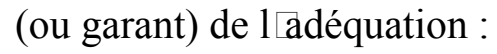

(12) J'aurais bien voulu vous amener mon mari. Malheureusement, il n'a pas pu venir

(13) Il faut réduire le nombre des fonctionnaires mais naturellement les services publics n'en seront pas affectés (Chirac, exemple analysé par E. Vladimirska dans sa communication au colloque sur le paradoxe, Pec, mars 2007).

Dans (12) : la présence de malheureusement présente $\mathbf{R}$ tel quill est exprimé par $\mathbf{p}$ comme une occurrence de malheur $^{31}$. $\mathbf{R}$ est donc représenté sur deux plans : comme 1Ґêvénement singulier qu est la non-venue, et comme relevant d un certain type dêvénement négatif. Dans (13) la présence de naturellement signifie que 1aabsence de conséquence négative liée à la réduction du nombre de fonctionnaires est inscrite dans «1 1 ordre du monde », ce qui tend à minimiser la dimension proprement événementielle de ce qui est dit; dans la bouche de Jacques Chirac, invoquer 1 ○rdre des choses vise surtout à dégager sa responsabilité dans ce qui peut se passer. Comme pour le cas précédent, $\mathbf{R}$ est représenté sur deux plans : comme un élément factuel (ce qu exprime p), et comme échappant aux contingences du factuel en tant quinscrit dans 1Øordre des choses. Dans ces deux exemples, le marqueur discursif formate 1 ẻvénement exprimé par $\mathbf{p}$ en le rapportant à un type dévénement.

\footnotetext{
${ }^{31}$ Cela n־est qu une formulation provisoire : dans une approche plus systématique il faudrait expliciter ce que recouvre « malheur ».
} 
Il est important de souligner qu une même base lexicale peut donner lieu à deux redéploiements sur le plan discursif : adéquation et point de vue. Cऍest le cas de pravda en russe (Paillard, 2002). En français, on peut donner comme exemple les paires en réalité / réellement et en effet / effectivement: la sémantique du point de vue est présente avec en réalité et en effet, celle de 1adéquation par réellement et effectivement (les exemples cidessous sont repris de Franckel \& Paillard, 2008) :

(14) Je ne sais pas quelle crainte au juste vous avez. Réellement (?? en réalité) je ne sais pas

(15) On aurait pu penser que pour une fois Paul était sincère. En réalité (?? réellement) il n'avait jamais autant trompé son monde.

(16) Réellement / en réalité vous ne m'aimez pas ....Adèle

Les MD participent des mécanismes généraux de construction du dire. Ci-dessus, dans la définition du dire, nous avons posé qu au départ il y a plusieurs séquences possibles, ce qui est noté $(\mathbf{p}, \mathbf{p})$, et que cette pluralité permet la prise en compte de 1aactivité de reformulation avec le passage de $\mathbf{p}$ à $\mathbf{p} \square$ La sémantique du point de vue peut être mise en relation avec le fait quau départ on pose $(\mathbf{p}, \mathbf{p})$ : on passe de $\mathbf{p}$ premier point de vue à $\mathbf{p}$ second point de vue. En revanche, la sémantique de 1adéquation signifie que p est en concurrence avec $\mathbf{p} \square$ pour ce qui est de rendre compte de $\mathbf{R}$.

Dans le cadre de cet article nous n aborderons pas le problème de la distribution des MD. Sur ce point, on peut se reporter à Franckel \& Paillard (2008).

\subsection{Les particules énonciatives}

Le terme de particules est parfois utilisé comme un terme générique pour désigner les marqueurs discursifs (cf. le titre de 1Øouvrage déjà cité Approaches to discourse particles ou encore le titre du numéro du Belgian Journal of Linguistics, 16, consacré aux marqueurs discursifs). Nous 1๔employons ici pour désigner une sous-classe qui possède une sémantique particulière : les particules ne participent pas à la spécification de $\mathbf{p}^{32}$ comme une façon

\footnotetext{
${ }^{32} \mathbf{p}$ désigne la séquence correspondant à la portée de la particule; cette portée est variable et est conditionnée par le fait quen russe les particules peuvent être proclitiques ou enclitiques. Sur ce point, cf. Bonnot \& Kodzassov 1998
} 
particulière dexprimer 1 état de choses $\mathbf{R}$. Elles spécifient $\mathbf{p}$ du point de vue de son rapport à

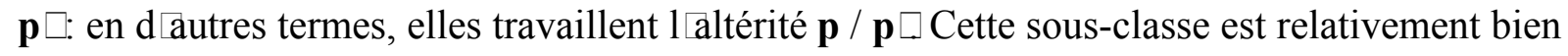
identifiée en russe et coïncide plus ou moins avec ce qui dans la tradition grammaticale russe est désigné par le terme časticy (« particules »). De même, en allemand, elles correspondent à ce qui est désigné par le terme Abtonungspartikeln ${ }^{33}$. En revanche, pour le français, où la notion de particule est étrangère à la tradition et à la terminologie grammaticale, línventaire des marqueurs discursifs ayant le statut de particules reste à faire ${ }^{34}$. Nous donnerons trois exemples empruntés au russe mettant en jeu les particules $u \check{z}$, ved' et bylo (sur la distribution de ces particules on peut se reporter à Bonnot \& Kodzassov 1998) :

$(17)=(3) \square$ Prideš $?$ ? - Da už pridu

- Tu viens ? $\square$ Oui je viens

(18) Čto ty tam sidiš’ celye dni. Ty im mešaes’. Ved $\square$ Saša tjaželo bolen

Qu âs-tu à rester là-bas des journées entières ? Tu les gênes. Ved $\square$ Sacha est gravement malade

(19) Ja vzjala bylo s soboj sigarety, a ix net

je prendre (perf.) bylo avec pron. Réf. Cigarettes et elles nég

Je croyais pourtant avoir pris mes cigarettes mais je ne les retrouve pas

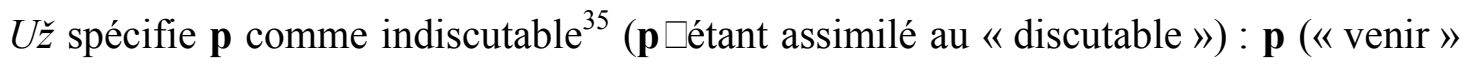
dans (17)) est spécifié comme débarrassé de toute forme daltérité dont $\mathbf{p} \square$ (« ne pas venir ») serait la trace («ne pas venir» signifie «venir est discutable»). En dautres termes, p incorpore $\mathbf{p} \sqsubset$ pour en signifier qu il n $₫$ a pas / plus de pertinence (cela n est pas sans rappeler la formule de Jean Luc Godard : «dans impossible il y a possible »). Dans (17) p «venir » est indiscutable au sens où So renonce à discuter $\mathbf{p}$ (ce qui signifie qu $a$ priori pour lui $\mathbf{p}$ est discutable).

Ved' confère à $\mathbf{p}$ le statut dun savoir objectif, par rapport auquel la seule extériorité est 1/ignorance $(\mathbf{p})$. Le recours au « $\mathbf{p} \llbracket$ savoir » est un moyen de dépasser un conflit présent dans le contexte gauche.

\footnotetext{
${ }^{33}$ Sur les particules de 1 allemand, cf. R. Mettrich et alii, Les invariants difficiles (4 volumes). Université de Metz.

${ }^{34}$ Sur la base des travaux de A. Culioli (1990) et P. Péroz (1991), bien dans des énoncés comme On achève bien les chevaux (pourquoi pas les hommes?) peut être rattaché à un fonctionnement particulaire.

${ }^{35}$ Sur $u z ̌$, cf. Paillard (2005) (comme la plupart des particules du russe $u z ̌$ ne se traduit pas).
} 
$B y l o^{36}$ signifie que, pour ce qui est du procès, on a la coexistence (ordonnée) de $\mathbf{p}$ et de

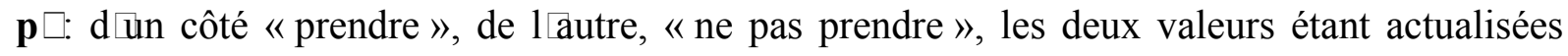
mais de façon décalée 1 une par rapport à 1 autre.

\subsection{Les mots du dire}

Cette désignation est empruntée à la thèse de E. Khatchaturian (2006) consacrée à quatre marqueurs discursifs du français et du russe formés avec le verbe dire (skazat' en russe) : pour ainsi dire, disons, tak skazat' (litt. ainsi + dire) et skažem (« disons »).

Nous proposons délargir cette sous-classe à dautres séries de marqueurs. Ils ont en commun le fait de marquer que la séquence correspondant à leur portée relève dun vouloir dire autonome distinct du vouloir+dire du locuteur, ce qui donne un dire « clivé ».En 2, en dehors du vouloir+dire du locuteur, nous avons introduit le vouloir dire des mots, c êst-à-dire ce quiils signifient en tant que tels, en dehors de leur utilisation dans un énoncé donné : 1‘idée est que les mots ne sont pas toujours des moyens fiables (sans parler du fait que 1 1 on ne trouve pas nécessairement « ses » mots) et le vouloir dire du monde (d un état de choses du monde) : un énoncé est toujours une représentation $\square$ interprétation de 1 êtat de choses quill exprime.

- pour ainsi dire : une partie des mots formant la séquence $\mathbf{p}$ (la portée de ces marqueurs est toujours «locale») est présentée comme autonomisée par rapport au vouloir + dire du locuteur : ce «vouloir dire » des mots est présenté comme nêtant pas pleinement adéquat. Les effets de sens vont de 1utilisation métaphorique du mot à différents effets rhétoriques : atténuation ou au contraire exagération.

(20) Deux nouveaux-nés dans la presse. Et pour ainsi dire jumeaux.

(21) J'ai, pour ainsi dire, une âme littéraire

- disons : p est présenté comme dire de compromis / dire sous toute réserve / dire en suspens / dire partagé. Dans le prolongement du statut premier dimpératif de la forme disons, la séquence $\mathbf{p}$ correspondant à la portée du marqueur (10 heures dans (22) ci-dessous) est mise en relation avec deux positions subjectives : celle de So avec la sélection de 10 heures / celle

\footnotetext{
${ }^{36}$ Sur bylo, on peut se reporter à la thèse de Tatiana Bottineau (2005), d où est tiré 1๔exemple (19).
} 
de S1 qui est compatible avec la sélection ou non de 10 heures, ce qui fait que la validation de 10 heures est représentée comme en suspens.

(22) Alors on se retrouve demain à la fac, disons à 10 heures

- mol, de, deskat' : 1eênoncé p est présenté comme la traduction-interprétation d un vouloir + dire dun sujet autre que le locuteur; (ce vouloir + dire étranger peut être présent dans le contexte gauche immédiat à travers un geste ou une autre forme de manifestation comme la référence à un dire non explicité), ce que $1\lceil$ on peut noter : vouloir + dire de Sx $\square$ mots de So (Sx désigne le sujet autre que So)

(23) Devuška otvetila suxo I nejtral'no : mol, ej voobšče vse ne nravitsja

La fille répondit $\mathrm{d}$ un ton sec et neutre: mol, de façon générale rien ne lui convenait (« $\square$ signifiant par là que $\square »)$

(24) Dokumenty na usynovlenie ona podavala četyre raza $i$ vse četyre raza ej otkazyvali : deskat, vy so svoej rabotoj ne smožete obespečit' mal'čiku nadležaščie uslovija žizni.

Elle avait déposé à quatre reprises une demande dadoption et à quatre reprises on avait rejeté sa demande : deskat $\square$ avec votre emploi vous ne pouvez pas garantir à 1匹enfant les conditions de vie convenables.

La différence entre mol et deskat' tient au fait que mol signifie que les mots utilisés sont le reflet fidèle du vouloir+dire de Sx (pondération sur le vouloir+dire) alors qu avec deskat' les mots utilisés sont une interprétation subjective de ce vouloir+dire (pondération sur 1ênoncé). Sur le plan prosodique, cela se traduit par le fait quavec mol on a une intonation neutre (caractéristique par ailleurs des propositions enchâssées) alors qu avec deskat' 1 intonation est marquée : les mots utilisés sont un enjeu pour So.

- brèves remarques sur le médiatif (évidentiel)

Nous avons vu quâvec mol et deskat', p est présenté comme la traduction-interprétation du vouloir+dire dun autre sujet. Nous faisons 1hypothèse quill est possible de rendre compte, dans des termes comparables, dun autre ensemble de données regroupées sous 1eetiquette de médiatif $^{37}$ (cf. en particulier la communication de Z. Guentchéva au colloque international $\mathrm{d}$ Anvers, «la notion de prise en charge en linguistique », 11-13 janvier 2007, et les

\footnotetext{
${ }^{37}$ Le terme de « médiatif » a d ailleurs été utilisé pour décrire des unités comme mol et deskat'.
} 
références bibliographiques qui figurent dans son exemplier). Avec le médiatif, 1 ênoncé p traduit / interprète un vouloir dire du monde (Guentchéva parle de raisonnement abductif à partir dindices). Nous donnons ci-dessous un exemple emprunté à Guentchéva :

(25) aman-a nipe rak o-kyt

Kamayura, Haut Xingu, Brésil

pluie Ntr Att 3 pleuvoir
Il a dî pleuvoir $\quad$ (indices : la route est mouillée, il y a des branches et des feuilles par terre)

p se présente comme 1 lênoncé matérialisant un vouloir dire externe (celui du monde) qui, à la différence des cas déjà envisagés, $\mathrm{n}$ est pas en tant que tel formulable : $\mathbf{p}$ dans ce cas revient à donner une forme linguistique à ce vouloir dire du monde, ce qui par définition revient à 1 iinterpréter. Ce travail dinterprétation peut se faire selon des modalités variables, en fonction, notamment, du mode daccès au monde (organes sensoriels comme la vue ou 1 ouïe dire, etc).

Comme pour les autres marqueurs discursifs, notre approche des mots du dire se limite ici à montrer comment il est possible dintégrer ces marqueurs dans la construction du dire : la sémantique de ces unités porte sur le fait que 1ểnoncé (ou fragment dểnoncé correspondant à la portée du marqueur discursif) est la mise en mots dun vouloir dire extérieur (vouloir dire dun sujet, des mots ou du monde). Au centre de ce travail énonciatif il y a un hiatus entre 1ênoncé et ce vouloir dire extérieur qui par définition ne peut qu excéder les mots qui lincarnent.

\section{Conclusion}

Dans cet article nous avons proposé une approche « intégrée » des phénomènes en jeu lorsqu un sujet produit un énoncé pour exprimer un état de choses du monde. Il est possible de résumer les principaux points sur lesquels notre approche diverge de celles qui utilisent la notion de prise en charge, $\mathrm{d}$ une part, celles qui mettent en jeu la notion de commitment.

Prise en charge : de notre point de vue 1 eenonciation n est pas uniquement une affaire de « sujet(s)» y compris assimilés à des voix (ou encore à des points de vue). Dire le monde (partant de son opacité première) est au c $\square$ ur du travail énonciatif, tout en se présentant comme une entreprise qui par définition ne peut être achevée.

Commitment : la notion de dire redéfinit la notion de contenu comme associant un « vouloir + dire » et un énoncé, sans quiil y ait de rapport nécessaire entre ce «vouloir + 
dire » et 1eẻnoncé qui le manifeste. Un dire défini comme une façon partiale et partielle d'exprimer par un énoncé un état de choses du monde est profondément subjectif et à ce titre échappe à une problématique du vrai / faux définie hors de 1๔espace du sujet. Nous ne pensons pas quill soit possible de construire une frontière stricte entre sémantique et pragmatique. En particulier, notre caractérisation des différents types dênoncés (assertion, injonction, question, exclamation) ne fait pas appel à la notion d acte illocutoire.

En positif, la notion de scène énonciative se présente comme un espace où, à partir des agencements de formes (y compris les marques prosodiques) constituant un énoncé, il est possible de restituer et les positions subjectives en jeu, et le statut du dire en tant que donnant une forme linguistique à un état de choses du monde. La scène énonciative se présente comme un espace dynamique, où, en fonction de déterminations multiples et hétérogènes, est représenté de façon infiniment variable mais régulière la rencontre entre un (des) sujet(s), des formes linguistiques et le monde. Les différentes classes de marqueurs discursifs peuvent être décrites comme contribuant (en fonction de leur sémantique propre) à la spécification du dire

\section{Bibliographie}

Bonnot, C., Kodzassov, S., 1998, «La variation sémantique des mots du discours et son influence sur la linéarisation et 1iintonation de 1 ënoncé »(en russe), in : Kisseleva, K. et Paillard D. (éds) Diskursivnye slova russkogo jazyka ( Les mots du discours du russe), izd. Metatekst, Moscou, pp. $382 \square 443$.

Beyssade, C., Marandin J.M., 2008, à paraître, « Commitment : attitude propositionnelle ou attitude dialogique »,

Bottineau, T., 2005, Etude de la particule énonciative bylo en russe contemporain, Thèse de doctorat, INALCO, Paris.

Carlson, G.N., 1978, Reference to Kinds in English, Bloomington, Indiana University Club.

Culioli, A., 1980, «Valeurs aspectuelles et opérations énonciatives », in : La notion d'aspect, Recherches linguistiques 5, Université de Metz, pp. 182 - 193.

Culioli, A., 1990, Pour une linguistique de l'énonciation I, Ophrys, Gap Paris, pp. $135 \square 169$.

Culioli A., 2001, «Heureusement!», Saberes no tempo - Homenagem a Maria Henriquez Costa Campos, Lisboa, pp. $279 \square 284$

Culioli, A., Paillard, D., 1987, «A propos de 1 alternance Imperfectif / Perfectif dans les énoncés impératifs », Revue des Etudes Slaves LIX : 3, Paris, pp. 527 - 534. 
De Voguë S., 1992, « Culioli après Benveniste : énonciation, langage, intégration », LINX 26, Université Paris X Nanterrepp. $77 \square 106$.

Danjou Flaux, N. Gary Prieur N., 1993, «Forcément ou le recours à la force dans le discours », Modèles linguistiques, III, 2, PU de Lille, Lille, pp. $54 \square 112$.

Dendale, P., Coltier, D., 2003, «Point de vue et évidentialité », Cahiers de praxématique 41, Université de Montpellier, pp. $105 \square 130$.

Dendale, P., Coltier, D., 2005, "La notion de prise en charge ou de responsabilité dans la théorie scandinave de la polyphonie linguistique", in J. Bres, P. P. Haillet, S. Mellet, H. Nølke \& Rosier, L., Dialogisme et polyphonie. Approches linguistiques, Bruxelles, De Boeck / Duculot, pp. 125-140

De Voguë, S., Paillard, D., 1987, « Modes de présence de 1autre », Particules énonciatives en russe contemporain 2, Collection ERA 542, Université Paris 7, pp. 11 - 37.

Ducrot, O.,1984, Le dire et le dit, Les Editions de minuit, Paris.

Fisher, K., (ed.), 2006, Approaches to discourse particles, Studies in pragmatics 1, Elsevier.

Franckel, J.J, Paillard D., 1997, «Représentation formelle des mots du discours : le cas de d'ailleurs », Revue de sémantique et de pragmatique, 1, PU d Orléans, pp. 51 - 69.

Franckel, J.J., Paillard, D., 1998 « Aspects de la théorie d Antoine Culioli », Langages, 129, pp. $52 \square 63$.

Franckel, J.J., Paillard, D., 2008, à paraître, « Mots du discours : adéquation et point de vue. L exemple de réellement, en réalité, en effet, effectivement», Conférence internationale Conectores e marcadores discursivos, Lisbonne, $2 \square 3$ novembre 2006.

Khatchatourian, E., 2006, Les mots du discours formés avec dire / skazat $\square$ en français et en russe, Thèse de doctorat, Université Denis Diderot $\square$ Paris 7

Kodzassov, S., 2003, "Varieties of focalisation in Russian : semantics and prosody », Mémoires de la Société de Linguistique de Paris, Nouvelle série, Tome XIII, pp. 99-113

Laurendeau, P., 1989, « Repérage énonciatif et valeur de vérité : la prise en compte et la prise en charge », in : Vincent, D., Saint jacques D. (dir.) Actes du CELAT, 2, Publications du CELAT, Université Laval, Québec, pp. $107 \square 129$.

Paillard, D., 1998, «Les mots du discours comme mots de la langue I », Le Gré des langues, 14, LHarmattan, pp. 10 - 41.

Paillard, D., 1999, Les mots du discours comme mots de la langue II », Le Gré des langues, 16, LHarmattan, pp. 99 - 115 
Paillard, D., 2002, "Pravda comme mot du discours" (en russe), in : K. Kisseleva et D. Paillard eds. Diskursivnye slova : opyt kontekstno-semantičeskogo analiza II ("Les mots du discours du russe : essai de description sémantico-contextuelle 2"), izd Russkie Slovari.

Paillard, D., 2005, « Towards a comparison of discursive markers », Third Conference on contrastive semantics and pragmatics, Shanghaï, septembre 2005.

Paillard, D., 2006a, « Discourse words in Russian », Sprache und Datenverarbeitung, 30.1, $69 \square 81$.

Paillard, D., 2006b « De la reconnaissance», in: D. Ducard, C. Normand (éds) Antoine Culioli. Un homme dans le langage $\square$, Ophrys, pp. $169 \square 183$.

Paillard, D., 2008a, « A propos des emplois non injonctifs de liimpératif en russe », in: R. Roudet, Ch. Zaremba (éds) Questions de linguistique slave. Etudes offertes à Marguerite Guiraud Weber, Presses de 1Université de Provence, pp. 217 - 234

Paillard, D., 2008b, à paraître, «Marqueurs discursifs et scène énonciative » PU de Normandie.

Péroz, P., 1991, Systématique des valeurs de bien, Librairie Droz, Genève.

Les invariables difficiles. Dictionnaire allemand français des particules, connecteurs, interjections et autres ,,mots de la communication“, I, II, III, IV, 1994 - 1998

Particules énonciatives en russe contemporain I, II et III, Institut des Etudes Slaves, 1986 1987.

Van der Wouden, Ton Foolen, Ad \& Van de Craen, Piet (eds), 2003, Particles, Amsterdam, John Benjamins Publishing Company. (Belgian Journal of linguistics, 16). 